\title{
Introduction to the special issue on enterprise architecture management
}

\author{
Robert Winter $\cdot$ Christine Legner $\cdot$ Kai Fischbach
}

Published online: 2 April 2013

(C) Springer-Verlag Berlin Heidelberg 2013

\section{Enterprise architecture management as a maturing discipline}

In the last decades, the complexity of corporate information technology (IT) environments has been growing steadily, and keeping pace with an ever-changing business environment has become a constant challenge. These factors have combined to make architecture a key concern of information management today. There is now general acknowledgment that only continuous and holistic management of the Enterprise Architecture (EA) can ensure the sustainability, agility, and strategic alignment of corporate IT environments.

Enterprise architecture has evolved as a discipline since the 1990s. Early work focused on architecture models, principles, and standards that comprise the content of the enterprise architecture. As companies have gained practical experience implementing EA concepts, they have become more concerned with enterprise architecture management (EAM) and its success factors.

Despite a growing body of EA-related knowledge, different definitions of EAM coexist, ranging from a narrow focus on enterprise-wide IT management to a broader focus on organizational development and transformation of the entire firm.

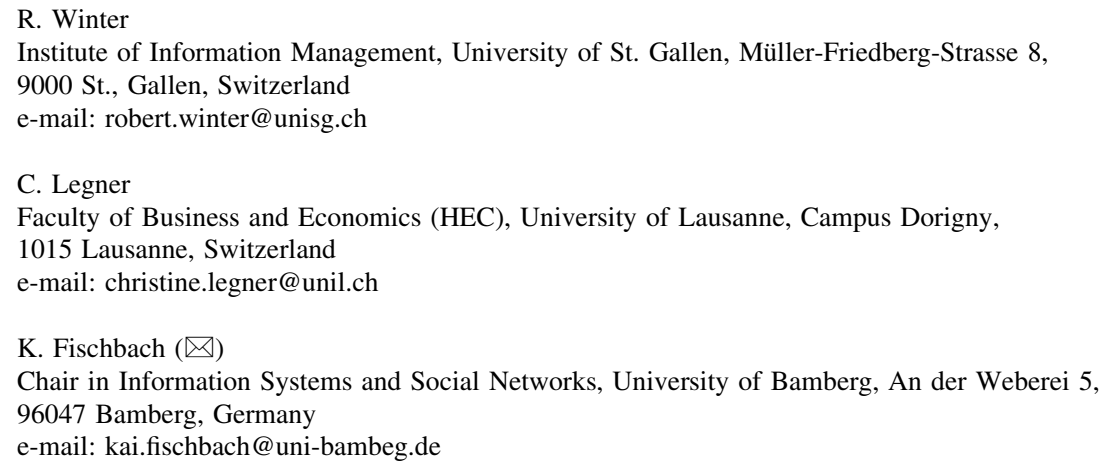


Correspondingly, researchers have identified substantial differences with respect to EAM objectives, methodology, and organizational implementation in practice. This has spurred studies of situational aspects of EAM implementation and fuller EAM adoption scenarios. Still, EAM is a rather young and immature discipline, characterized by a great variety of research approaches and a strong focus on conceptual frameworks and models. Until recently, theoretically grounded research on EAM practice has been quite limited.

\section{Current status and trends in enterprise architecture management}

With this special issue, the Journal of Information Systems and e-Business Management seeks to present the current state of EAM research. This state includes, but goes far beyond, conceptualizing EA (e.g., with meta models, roadmaps, or specific architectures such as business architecture), and designing EAM models, tool support, and EAM-related standards.

Although the basics of EA management are covered by investigating EAM organization and governance (e.g., processes, organizational roles, monitoring and KPIs), current managerial considerations should also include the strategic aspects of EAM, such as positioning and goals, services and "products", communication, and stakeholder involvement. The shift from "technical" to "managerial" topics in EAM implies consideration of specific EAM application contexts (e.g., M\&A, business transformation, outsourcing, IT/business alignment) and the organizational implementation of EAM (e.g., situational adaptation of EAM). As with any other information management sub-discipline, EAM is increasingly reflecting research in adjacent management disciplines, notably strategic management, organizational development, and IT management. Similarly, research in IS adoption, success, and value is beginning to incorporate EAM-related issues. Finally, EAM research is always inspired by innovative cases because, ultimately, IS research problems arise in the real world, and IS research results should create real-world value.

\section{Contributions to this special issue}

The four papers in this special issue address the trends in EAM research just described. They cover "classical" topics such as service-oriented architectural design, but provide a new spin on how these topics are dealt with, emphasizing their adoption in practice. The papers also introduce new strategic and organizational perspectives by focusing on business-oriented EAM (business architecture and strategic application scenarios), the impact of organizational culture on EAM effectiveness, and the embedding of EAM in IT management.

Simon, Fischbach, and Schoder explore EAM's role in corporate strategic management. Their exploration is grounded in a comprehensive framework of business architecture content, which they use to help grasp what constitutes the business part of enterprise architecture. The authors identify different strategic management scenarios in which they analyze of use of EAM, such as strategic 
analyses, strategic choice, business transformation readiness assessments, and strategy implementation planning. Their empirical grounding comes from interviews with strategic management practitioners, whose results support the use of enterprise architecture as a frame of reference for strategic management. The paper makes a significant contribution to both academia and practice; with their work, the authors offer novel insights into how to leverage EA models way beyond management of the IT landscape.

Aier has chosen organizational culture as an aggregated construct to describe different EAM situations. He focuses on the role of organizational culture in effectively anchoring EA artifacts within organizations. Specifically, he analyses how organizational culture influences the effectiveness of enterprise architecture principles (EAP). His research, based on empirical data, shows that organizational culture significantly influences the way EAP grounding, management, and EAP guidance affect EA consistency and EAM utility. Aier makes recommendations, based on his findings, for dealing with selected design decisions when introducing and developing EA principles appropriate to different cultural settings within organizations, and shows where managers should invest limited resources to make EAP effective.

Van Belle and MacLennan investigate the adoption of service-oriented architecture, based on an empirical study conducted among South African IT professionals. Their work is highly relevant both for academia and practice, since it focuses on one of the most prominent architectural styles and contributes quantitative empirical insights to a field still dominated by qualitative studies. The authors combine two theoretical lenses, the diffusion of innovation theory and the technology-organization-environment (TOE) framework, to uncover relevant factors affecting SOA adoption. From their empirical study, they find that top management support, governance, resources, and vendor support are significant factors in addition to architectural choices and EA compatibility, both for both SOA adoption and for (perceived) SOA project success.

Löhe and Legner's research is motivated by the many failures and severe challenges companies face in implementing EAM. They suggest that EAM should not replace existing IT management practices, but needs to complement and enhance those practices. Basing their work on case studies, the authors first systematically analyze design requirements and then suggest a design theory for the implementation of enterprise architecture management, which they call architecture-driven IT management (ADRIMA). Their main contribution lies in developing prescriptive knowledge for embedding enterprise architecture management into existing IT management processes and structures, particularly their seven design principles.

The articles in this special issue underpin that EAM research has matured over the past several years. While modeling and even organizational basics are assumed to be sufficiently diffused, the interest shifts towards more "difficult" EAM topics and a better theoretical grounding. The articles illustrate the role and contribution of EAM in achieving management goals such as flexibility (rather than simply providing transparency), in the application of EAM in strategic management (that is, beyond software solution development), and in the context of organizational culture. 


\section{Outlook}

While predictions are always difficult to make, some assumptions about the future of EAM research seem reasonable. As more and more sub-topics become better understood, the consensus regarding key concepts in EAM will grow. This will result in "dominant designs" or even standardization, both with respect to the conceptualization of the architecture (e.g., architectural layers and elements, reference models) as well as the EA development and lifecycle (e.g., The Open Group Architectural Framework, TOGAF). With more clear definitions and foundations, EAM research can move from deployment-related topics to use/ value-related topics such EAM adoption and adaptation in specific project or organizational contexts, or the acceptance, effectiveness, and value of EAM as a strategic management approach.

Ultimately, this will lead to more differentiated research, from both a design and an explanatory perspective. As mainstream EAM matures even more, it will contribute to more effective and efficient EAM implementation in practice.

There are several offsprings emerging from EAM. These include:

- strategic transformation support (EAM + strategic controlling + other enterprise-wide resource management functions);

- strategic management support (inclusion of architectural thinking in managerial decision making); and

- "lightweight" EAM (fast architectural support for urgent business questions).

We believe architectural support for complex decisions in the context of people, organization, and technology will remain an important and innovative field of IS research for quite some time.

Acknowledgments We gratefully acknowledge the support and assistance of the Associate Editors Frederik Ahlemann, João Paulo A. Almeida, Brian Cameron, Marijn Janssen, Robert Lagerström, Susanne Leist, Florian Mattes, Erik Proper, Raymond Slot, and José Tribolet. 